\title{
ACTITUDES Y CONDUCTAS SEXUALES DE RIESGO PARA LA INFECCIÓN POR VIH/SIDA EN JÓVENES ESPAÑOLES
}

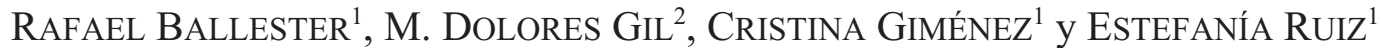 \\ ${ }^{1}$ Universidad Jaime I de Castellón \\ ${ }^{2}$ Universidad de Valencia
}

\begin{abstract}
Resumen: En este estudio se analizan las actitudes y conductas sexuales de riesgo de los jóvenes españoles respecto a la infección por VIH. Se administró el Cuestionario de Prevención del SIDA a 196 jóvenes. Los resultados muestran lagunas importantes en la información (sólo el 59\% sabe que el sexo oral sin preservativo es una práctica de riesgo); el uso sistemático del preservativo es moderado en el coito (indican haberlo utilizado en el 53\% de las relaciones del mes anterior); y muy bajo en las relaciones orales $(3 \%)$ y anales (13\%), mantenidas por un elevado número de sujetos (el $73 \%$ realizan prácticas orales y el $16 \%$ anales). Hay una baja percepción de peligro lo que se manifiesta en que sólo el $14 \%$ preguntan a sus parejas si se han realizado las pruebas del VIH y tan sólo el $40 \%$ se las ha realizado alguna vez. Urgen intervenciones preventivas en este colectivo.
\end{abstract}

Palabras clave: Infección por VIH, conductas de riesgo, jóvenes

Attitudes and risk sexual behaviors for HIV infection/AIDS in young people in Spain

\begin{abstract}
This study analyzes attitudes and sexual risk behaviors of young people in Spain with regard to HIV infection. The AIDS Prevention Questionnaire was administered to 196 subjects. The results evidence important gaps in information (only 59\% knows that oral sex without a condom is a risk behaviour); the systematic use of a condom is moderate in intercourse (in 53\% of sexual intercourse over the past month) and very low in oral (3\%) and anal (13\%) sex, as practiced by a large number of subjects ( $73 \%$ do oral sex and $16 \%$ anal sex). There is a low sense of danger as manifested by the fact that only $14 \%$ ask their sexual partners if they did any HIV testing and only $40 \%$ have passed a HIV test at any time. Preventive interventions are urgently needed in this population.
\end{abstract}

Keywords: HIV infection, risk behaviors, young people.

\section{INTRODUCCIÓN}

La infección por VIH/SIDA sigue constituyendo en la actualidad uno de los principales problemas de salud pública en todo el mundo, que produce en las personas que lo padecen no

Recibido: 3-enero-2009; aceptado: 29-marzo-2009.

Correspondencia: Rafael Ballester, Facultad de Ciencias Humanas y Sociales, Universidad Jaime I, Avda. Sos Baynat, s/n, 12071 Castellón. Correo-e: rballest@psb.uji.es

Este estudio ha sido realizado en parte gracias a las ayudas de la Universidad Jaime I-Fundación Bancaixa (P11B2004-18 y P1.1B 2006-19) y de la Fundación para la Investigación y Prevención del SIDA en España (FIPSE) (Exp. 12436/03) sólo un alto grado de sufrimiento físico, sino también importantes alteraciones emocionales (Ballester, 2005; Edo y Ballester, 2006) agravadas entre otros factores por la exclusión social a la que se condena a estos enfermos (Flores, Borda y San Gregorio, 2006). Hoy por hoy, aparte de los grandes avances médicos que permiten a través de los fármacos antirretrovirales mejorar la calidad de vida de los enfermos, la única vía para luchar contra esta grave enfermedad es la prevención (Piña y Robles, 2005).

A pesar de su importancia, en los últimos años se ha documentado suficientemente el escaso uso del preservativo en las relaciones sexuales como método de prevención de la infección por VIH. Por citar algunos ejemplos, Gurman y Borzekowski (2004) han hallado que 
tan sólo el $41 \%$ de los estudiantes universitarios latinos de los Estados Unidos utilizaron el preservativo en su última relación vaginal y el porcentaje aún era más bajo en el sexo anal $(28 \%)$ y las relaciones orales (5\%). En nuestro contexto los resultados van en la misma línea (Ballester, Gil, Edo y Giménez, 2007; Ballester, Gil, Giménez, Edo y Gil, 2007). Otros estudios evidencian importantes lagunas informativas entre los jóvenes (Ballester, Gil, Gómez y Edo, 1999; Farid y Choudhry, 2003). Su confianza en el preservativo como método de prevención no es elevada (Duncan, Miller, Borskey, Fomby, Dawson y Davis, 2002, Giménez, Ballester, Gil y Edo, 2006) pero sobre todo su percepción del riesgo de infectarse es escasa (Planes, Gómez, Gras, Font-Mayolas, Cunil, Aymerich y Soto, 2006), especialmente en las relaciones que consideran estables.

Con el fin de conocer los factores contribuyentes que explican los comportamientos de riesgo en los jóvenes se ha intentado aplicar modelos clásicos como el de Creencias de Salud (Winfield y Whaley, 2002), la teoría de la Acción Razonada (Bosompra, 2001), el Modelo de Autorregulación o el Modelo de Conducta de Salud de Morin (Ford, 1995). Las investigaciones fundamentadas en estos modelos han obtenido evidencia empírica de la importancia de aspectos como la cooperación de la pareja sexual en la práctica del sexo seguro (Wilkinson, Holahan y Drane-Edmundson, 2002), el miedo al SIDA (Allison y Albarracín, 2007), aspectos situacionales de la interacción sexual (Von Sadovsky, Keller y McKinney, 2002), el consumo de alcohol antes o durante la relación sexual (Higgins, 2005; Gullette y Lyons, 2005) y de cannabis (Brodbeck, Matter y Moggi, 2006), las creencias acerca de la gravedad de la enfermedad (Demmer y Caroleo, 2001), las habilidades para el uso del preservativo (Lindemann y Brigham, 2003), las barreras percibidas para su utilización (Winfield y Whaley, 2002), la norma percibida en el grupo de iguales (Scholly, Katz, Gascoigne y Holck, 2005), la búsqueda de sensaciones (Gullette y Lyons, 2005) y la autoeficacia (Mashegoane, Moalusi, Ngoepe y Peltzer, 2004; Heeren, Jemmot, Mandeya y Tyler (2007); Ballester, Gil, Edo y Ruiz, 2007).
En resumen, estos resultados muestran la complejidad de factores que pueden explicar las conductas de riesgo para la transmisión sexual del VIH entre los jóvenes y alertan acerca de la necesidad de conocer estos factores con el fin de elaborar con urgencia programas preventivos dirigidos a este colectivo. Nuestro objetivo en este trabajo es aportar información exhaustiva y actualizada acerca de estos factores a través del análisis de los comportamientos y actitudes de riesgo de los jóvenes españoles.

\section{MÉTODO}

\section{Diseño}

Esta investigación se enmarca dentro de una acción más amplia iniciada en febrero de 2003 con la apertura de la Unidad de Investigación sobre Sexualidad y SIDA (UNISEXSIDA) en la Universidad Jaime I de Castellón, que incluye entre sus acciones la información directa a estudiantes, el desarrollo de talleres de prevención del SIDA, la existencia de un teléfono de atención al estudiante, la creación de una página web para consultas a través de internet y la aplicación individual y grupal de programas de intervención psicológica para los afectados por la enfermedad.

Los resultados que aportamos forman parte de un proyecto en el que tras evaluar a los jóvenes, se analizaba la eficacia de distintas estrategias preventivas sobre las actitudes y conductas de riesgo frente al VIH. El diseño de este proyecto más amplio es de comparación entre grupos con grupo control, si bien, como hemos dicho, aquí presentamos los primeros datos descriptivos resultantes de la evaluación antes de la intervención.

\section{Participantes}

La captación de los jóvenes se realizó dentro del ámbito de la Universidad Jaime I de Castellón y la Universidad de Valencia. A través de carteles informativos y del correo electrónico se ofreció a los estudiantes la posibilidad de participar en talleres de prevención del SIDA. Con el fin de que la muestra no estuviera ses- 
gada incluyendo tan sólo a jóvenes sensibilizados respecto a la problemática del VIH, se ofreció una pequeña gratificación económica ( 15 euros) a los participantes en el estudio. Los estudiantes cumplimentaron el cuestionario inmediatamente antes de comenzar la intervención preventiva de manera completamente confidencial y anónima.

Dado que al introducir los datos de los cuestionarios decidimos eliminar algunos cuyas respuestas no parecían demasiado fiables por ser contradictorias, de los 196 sujetos evaluados hemos analizado los datos referidos a 186 estudiantes universitarios. El 49,2\% de los estudiantes eran de la universidad de Castellón y el $50,8 \%$ de la de Valencia. El 27.8\% fueron hombres y el $72,2 \%$ fueron mujeres. La edad media fue de 22,28 años $(D T=4,36)$.

\section{Instrumento de evaluación}

La evaluación se llevó a cabo a través del Cuestionario de Prevención del SIDA de Ballester, Gil, Guirado y Bravo (2004), que incluye un total de 65 ítems de diferente formato referidos tanto a aspectos de información acerca de la infección por VIH, sus vías de transmisión y los métodos preventivos, como a actitudes, intención de conducta y comportamientos preventivos. La fiabilidad de este cuestionario evaluada a través de su consistencia interna (alfa de Cronbach) es de 0,70, su estabilidad temporal (fiabilidad test-retest) es de 0,83 y su validez convergente evaluada a través de su correlación con la Encuesta sobre el SIDA de Ballester y Gil fue de 0,79 (Ballester, Gil y Giménez, 2007a,b).

\section{RESULTADOS}

\section{Caracterización del perfil general de los participantes}

Respecto a la historia sexual general (ver Tabla 1), el 85\% de la muestra había mantenido en alguna ocasión relaciones sexuales con otra persona. La media de personas con las que habían mantenido relaciones sexuales fue de 4,48
$(D T=6,43)$. Un $7 \%$ había tenido alguna vez relaciones con personas de su mismo sexo. En lo que se refiere a la orientación sexual, el $91,6 \%$ de los sujetos se consideraban heterosexuales, el $4,5 \%$ bisexuales y el 3,9\% homosexuales. El $64 \%$ de la muestra mantenía en el momento de la evaluación relaciones con una pareja estable. El 10,5\% de los sujetos reconocieron haber sido infieles en alguna ocasión a su pareja estable y un $36 \%$ de los que lo hicieron manifestaron no haber utilizado el preservativo en esa relación esporádica.

Por lo que se refiere a la historia de comportamientos de riesgo (ver Tabla 1), el 29\% de los sujetos creía haber realizado alguna práctica de riesgo (una media de 7 veces). El 88,5\% de los que contestaron afirmativamente reconocieron que cuando lo hicieron disponían de información sobre las vías de transmisión del VIH y el 52\% llegaba a afirmar que eran conscientes en ese momento del riesgo que corrían. Es de subrayar que, sin embargo, sólo el $28 \%$ estuvo preocupado durante algún tiempo por la posibilidad de haberse infectado. Y aún más que tan sólo el $17 \%$ fue a realizarse las pruebas diagnósticas.

Finalmente quisimos saber el grado de importancia que tenía la salud para los estudiantes de la muestra. En una escala de 0 a 10, la media del grupo total fue de 9,45 $(D T=0,90)$, es decir, muy alta.

\section{Información sobre la prevención del SIDA}

Por lo que respecta a la información de que disponían los sujetos de la muestra, el 90\% manifestó haber recibido en alguna ocasión información sobre el SIDA. La fuente de la información fue principalmente las campañas informativas (74\%) seguidas por los profesores (56\%). Sólo el 23\% había recibido información de sus padres.

Tan sólo el 4,3\% de la totalidad de la muestra valoró su grado de información sobre la prevención del SIDA como "muy bueno", el 58\% valoraba este grado como "bueno", el 36\% como "regular" y el 1,6\% como "malo" (ver Tabla 2). Para evaluar el grado real de información, incluimos en el cuestionario un conjunto de ítems que exploraban el conocimiento acerca de las vías de 
Tabla 1. Perfil general de la muestra e historia sexual de las prácticas de riesgo

\begin{tabular}{lll}
\hline Ha mantenido relaciones con otra persona & $85 \%$ \\
Ha mantenido relaciones con personas del mismo sexo & & $7,3 \%$ \\
En cuanto a orientación sexual se considera: & Heterosexual & $91,6 \%$ \\
& Bisexual & $4,5 \%$ \\
Mantiene relaciones con una pareja estable & Homosexual & $3,9 \%$ \\
Tiempo de relación en meses & & $64 \%$ \\
Alguna vez ha sido infiel a su pareja estable & Media $=36,4(D T=28,9)$ \\
No usó preservativo en esta relación & $10,5 \%$ \\
Cree haber realizado alguna práctica sexual de riesgo & $36,4 \%$ \\
Tenía información cuando lo hizo & $29,4 \%$ \\
Era consciente del riesgo & $88,5 \%$ \\
Estuvo preocupado por la posibilidad de haberse infectado & $52,4 \%$ \\
Se hizo las pruebas diagnósticas & $28 \%$ \\
Importancia que tiene la salud de 0 a 10 & $16,7 \%$ \\
\hline
\end{tabular}

transmisión del VIH, de las prácticas sexuales de riesgo o de otros aspectos especíicos como la existencia del período ventana. Los resultados revelan en general un grado de conocimiento bastante alto entre la mayoría de los estudiantes, si bien también encontramos importantes lagunas, siendo de destacar las que se refieren al sexo oral (sólo un $59 \%$ cree que es una práctica de riesgo cuando no se utiliza el preservativo), al período ventana (sólo un $24 \%$ conoce su existencia), o al significado de las pruebas diagnósticas (sólo el $52 \%$ sabe que un resultado positivo no significa que se vaya a desarrollar necesariamente el SIDA con posterioridad).

\section{Percepción de riesgo y percepción de gravedad del SIDA}

La probabilidad percibida media de infectarse con el VIH en una escala de 0 a 100 era de 20,9 para los estudiantes universitarios (ver Tabla 3). Esta probabilidad fue algo menor de la probabilidad percibida de un embarazo no deseado, que fue del 24,4. Respecto al temor ante la posibilidad de infectarse con el VIH fue de 66,4, sólo algo superior al temor ante un embarazo no deseado (Media $=60,4$ ). También quisimos evaluar en qué medida se atribuía el SIDA a determinados "colectivos o grupos de riesgo" como una medida indirecta del riesgo percibido. Los estudiantes universitarios consideraban que el $55 \%$ de los afectados eran toxicómanos, el $24 \%$ homosexuales y el $21 \%$ heterosexuales.

Respecto a la percepción de gravedad (ver Tabla 3), el $0,5 \%$ de la muestra consideraba que el SIDA era una enfermedad leve, el $1 \%$ moderada, el $49,5 \%$ grave y el $48,9 \%$ fatal o mortal. Destaca que tan sólo el $73,7 \%$ conociera que el SIDA no tiene curación.

\section{Realización de pruebas de detección de anticuerpos y uso del preservativo en distintos tipos de relación y prácticas sexuales}

Ante la pregunta de si alguna vez se habían realizado las pruebas de detección de anticuerpos, sólo el $40 \%$ contestó afirmativamente (ver Tabla 3). Cuando preguntamos por la intención de volver a hacérselas, sólo el 19\% de la muestra afirmó tener esa intención. Consideramos importante también evaluar si los estudiantes sabían dónde realizarlas. Sólo el $46 \%$ de la muestra sabía dónde las podía hacer. Finalmente, únicamente el 14\% afirmó que solía preguntar a sus parejas si se habían realizado las pruebas diagnósticas. 
Tabla 2. Grado de información percibido y real sobre la prevención del SIDA

\begin{tabular}{|c|c|c|}
\hline \multirow[t]{4}{*}{ Tu grado de conocimiento sobre la prevención del SIDA es } & Muy bueno & $4,3 \%$ \\
\hline & Bueno & $57,8 \%$ \\
\hline & Regular & $36,3 \%$ \\
\hline & Malo & $1,6 \%$ \\
\hline ¿A simple vista puede saberse si una persona es portadora del virus del SIDA? (No) & & $90,9 \%$ \\
\hline $\begin{array}{l}\text { ¿Una persona afectada por el VIH puede transmitir la infección aunque no } \\
\text { presente síntomas? (Sí) }\end{array}$ & & $89,7 \%$ \\
\hline $\begin{array}{l}\text { ¿La mayoría de personas con SIDA muestran síntomas de estar enfermos } \\
\text { enseguida? (No) }\end{array}$ & & $87,1 \%$ \\
\hline ¿Puedo ser infectado por el VIH si practico únicamente sexo oral? (Sí) & & $56,8 \%$ \\
\hline La marcha atrás es un método fiable para prevenir la transmisión del VIH (No) & & $95,2 \%$ \\
\hline La píldora protege a la mujer del contagio del VIH (No) & & $93,5 \%$ \\
\hline El VIH es tan pequeño que puede pasar a través del condón (No) & & $81 \%$ \\
\hline Dar positivo en las pruebas implica desarrollar el SIDA con posterioridad (No) & & $52,2 \%$ \\
\hline ¿Conoces la existencia de un período ventana...? (Sí) & & $24,3 \%$ \\
\hline ¿Dirías que actualmente el SIDA es una enfermedad que tiene curación? (No) & & $73,7 \%$ \\
\hline \multirow[t]{12}{*}{ ¿Cuáles de las siguientes son vías de transmisión del VIH?: } & Vasos o cubiertos (no) & $87,1 \%$ \\
\hline & Agujas para jeringuillas (sí) & $98,9 \%$ \\
\hline & Cepillo dientes/cuchilla afeitar & $89,2 \%$ \\
\hline & Sangre (sí) & $100 \%$ \\
\hline & Sudor (no) & $71,9 \%$ \\
\hline & Flujo vaginal (sí) & $93 \%$ \\
\hline & Semen (sí) & $96,8 \%$ \\
\hline & Líquido preseminal (sí) & $73,5 \%$ \\
\hline & Saliva (no) & $73,7 \%$ \\
\hline & Leche materna (sí) & $42,2 \%$ \\
\hline & Agujas (piercings...) (sí) & $90,9 \%$ \\
\hline & Picadura de mosquito (no) & $34,9 \%$ \\
\hline \multirow{4}{*}{$\begin{array}{l}\text { ¿Con cuál de estas prácticas se puede transmitir el VIH si no se utiliza } \\
\text { preservativo?: }\end{array}$} & Masturb. mutuas (No) & $77,8 \%$ \\
\hline & Sexo oral (Sí) & $58,6 \%$ \\
\hline & Coito vaginal (Sí) & $98,9 \%$ \\
\hline & Coito anal (Sí) & $85,5 \%$ \\
\hline
\end{tabular}

Por lo que se refiere al uso del preservativo (ver Tabla 3), comentaremos en primer lugar, el tipo de prácticas sexuales más frecuentes entre los estudiantes universitarios, dado que este dato puede proporcionarnos algún tipo de información sobre la potencial situación de riesgo. Cabe señalar que el $67 \%$ de los estudiantes manifestaban practicar la masturbación, un $81 \%$ realizaban masturbaciones mutuas, un $80 \%$ practicaban el coito vaginal, $73 \%$ sexo oral y $16 \%$ coito anal. Es de destacar, sin embargo, que cuando preguntamos por el porcentaje de relaciones sexuales en que habían utilizado el preservativo en el último mes, éste fue del 53\%. A pesar de este bajo porcentaje referido al uso real del preservativo, la probabilidad estimada de utilizar el preservativo en las próximas relaciones fue de 87 (0 a 100). Al preguntar por la frecuencia real de uso del preservativo en distintas prácticas nos encontramos con que sólo 
utilizaban "siempre" el preservativo en el coito vaginal el $48 \%$, en el sexo oral el $3 \%$, en el coito anal el 13\%, en las relaciones con la pareja estable el $43 \%$ y en las relaciones esporádicas el $55 \%$. Destaca, por tanto, el bajo índice de uso del condón en las relaciones orales y anales. Finalmente preguntamos por el tipo de comportamiento que se llevaría a cabo en el caso de que surgiera posibilidad de una relación y no se tuviera preservativo. A este respecto, un 19\% afirmó que mantendría la relación pero evitando prácticas de riesgo, otro $19 \%$ se abstendría y el $69 \%$ intentaría conseguir un preservativo.

Ventajas e inconvenientes asociados al uso del preservativo, confianza en el preservativo como método de prevención, autoeficacia y norma percibida

Un $28 \%$ de la muestra no encontraba ninguna dificultad para el uso del preservativo (ver

Tabla 3. Percepción de riesgo, percepción de gravedad del SIDA, pruebas diagnósticas y uso del preservativo

\footnotetext{
Probabilidad percibida de infectarse con el VIH (0-100)

Probabilidad percibida de embarazo no deseado (0-100)

Temor ante la posibilidad de infectarse con el VIH (0-100)

Temor ante la posibilidad de embarazo no deseado (0-100)

Distribución de afectados por colectivos (Medias)
}

Dirías que el SIDA es una enfermedad:

Frecuencia de uso sistemático (siempre) de preservativo:

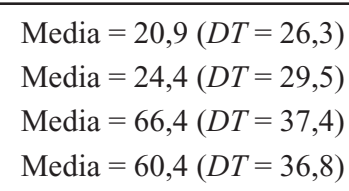

Media $=87(D T=23,8)$

Si te surgiera posibilidad de relación y no tuvie- Tendrías la relación exactamente igual $\quad 3,2 \%$ ras condón: ticas de riesgo 
Tabla 4). En cuanto al tipo de dificultad más importante aducido por los estudiantes cabe destacar la disminución de la sensibilidad (37\%), miedo a una posible rotura o mal estado del preservativo $(33 \%)$, interrupción de la relación (30\%), dificultad para obtenerlos $(25 \%)$, el precio (18\%), incomodidad (13\%) y la vergüenza al comprarlos $(9,7 \%)$ entre otros factores. Por el contrario, entre las principales ventajas asociadas al uso del preservativo destacan que "no tienen efectos secundarios" $(47 \%)$, "son efectivos para la prevención del SIDA y otras ETS" (93\%), "para la prevención de embarazos" (83\%), "son fáciles de conseguir" (51\%) y “son higiénicos" (30\%).
Un $52 \%$ de la muestra consideraba que el preservativo era un método "muy fiable" de prevención del VIH, un $41 \%$ un método "bastante fiable", un $6,5 \%$ "algo fiable" y un $0,5 \%$ "nada fiable" (ver Tabla 4). Como un índice global de autoeficacia en el uso del preservativo, preguntamos a los estudiantes si creían saber cómo utilizarlo, obteniendo una media de 83 en una escala de 0 a 100 . Finalmente, exploramos la valoración por parte de las personas significativas de su entorno acerca del uso del preservativo. A este respecto, un $98,4 \%$ afirmó que el uso del condón estaba bien visto entre la mayoría de gente importante para él/ella.

Tabla 4. Barreras y ventajas asociadas al uso del preservativo, autoeficacia y norma percibida

\begin{tabular}{|c|c|c|}
\hline \multirow[t]{12}{*}{ Barreras percibidas para el uso del preservativo } & Ninguna & $28 \%$ \\
\hline & El precio & $17,7 \%$ \\
\hline & Dificultad para obtenerlos & $24,7 \%$ \\
\hline & Interrupción de la relación & $30,1 \%$ \\
\hline & Menor sensibilidad & $36,6 \%$ \\
\hline & Falta de confianza en su eficacia & $8,1 \%$ \\
\hline & Incómodo & $12,9 \%$ \\
\hline & Vergüenza al comprarlos & $9,7 \%$ \\
\hline & Da corte ponérselos & $2,2 \%$ \\
\hline & Posible rotura o mal estado & $32,8 \%$ \\
\hline & Es una muestra de poca confianza con la pareja & $1,6 \%$ \\
\hline & Pueden pensar que siempre estás buscando rollo & $7 \%$ \\
\hline \multirow[t]{8}{*}{ Ventajas percibidas } & Es efectivo para la prevención de SIDA y ETS & $93 \%$ \\
\hline & Es fácil de conseguir & $51,1 \%$ \\
\hline & $\begin{array}{l}\text { Da juego en la relación sexual al poder elegir entre } \\
\text { sabores, formas... }\end{array}$ & $5,4 \%$ \\
\hline & Es eficaz para la prevención de embarazos & $80,6 \%$ \\
\hline & Su uso es práctico & $23,1 \%$ \\
\hline & Bajo coste económico & $9,7 \%$ \\
\hline & No tiene efectos secundarios & $46,8 \%$ \\
\hline & Es más higiénico & $30,1 \%$ \\
\hline \multirow{4}{*}{$\begin{array}{l}\text { ¿Hasta qué punto consideras que el preservativo es } \\
\text { un método fiable de prevención del SIDA? }(\%)\end{array}$} & Nada & $0,5 \%$ \\
\hline & Algo & $6,5 \%$ \\
\hline & Bastante & $40,9 \%$ \\
\hline & Mucho & $52,2 \%$ \\
\hline $\begin{array}{l}\text { ¿Hasta qué punto crees que sabes cómo utilizar el } \\
\text { preservativo en tus relaciones? }(0-100)\end{array}$ & & $\begin{array}{l}\text { Media }=83 \\
D T=18\end{array}$ \\
\hline $\begin{array}{l}\text { Entre la mayoría de gente importante para ti está bien } \\
\text { visto el uso del condón (Sí) }\end{array}$ & & $98,4 \%$ \\
\hline
\end{tabular}




\section{Solidaridad hacia los afectados por la enfermedad}

Sólo un $17 \%$ de los estudiantes conocían a una persona infectada por el VIH (ver Tabla 5). El grado de sufrimiento que los estudiantes creen que experimenta un enfermo con VIH es elevado (Media $=86,9, D T=15,6$, en una escala entre 0 y 100) y también el grado en el que consideran que la sociedad debería ser más comprensiva y solidaria con los enfermos con VIH (Media $=92, D T=14,2$ en una escala entre 0 y 100). El 94,6\% de los universitarios cuidaría de un amigo que tuviera el SIDA (ver Tabla 5). También un $74 \%$ de la muestra total afirmó que si un amigo fuera portador del VIH intentaría quedar con él para ayudarle, un $25 \%$ se comportaría exactamente igual que antes y sólo un $2,1 \%$ evitaría verle frecuentemente o dejaría de verle por completo. Ante la pregunta de qué harían si antes de conocer a alguien se enterasen de que es portador del VIH, el 87,6\% afirmó que lo conocería exactamente igual, un 5,4\% que tendría incluso más interés en conocerle, un $17,3 \%$ informaría a sus amigos para que le apoyaran más y sólo un 1,6\% y 2,2\% respectivamente evitaría conocerlo o alertaría a los amigos para que se preservaran.

\section{DISCUSIÓN}

Los resultados de nuestro estudio muestran una situación preocupante en el colectivo de estudiantes universitarios en lo que respecta a la prevención del SIDA. A pesar de que la mayoría de estudiantes manifiestan haber recibido en alguna ocasión información sobre el SIDA, sobre todo a partir de las campañas informativas, son muchas las lagunas de información que mantienen los estudiantes, al igual que se observa en otros estudios como el de Farid y Choudhry (2003), especialmente por lo que se refiere al sexo oral, al período ventana y al significado de las pruebas diagnósticas. De hecho, un $38 \%$ de los estudiantes reconocen su grado de información como regular o malo.

Por otro lado, al igual que se destaca en otros estudios como el de Planes et al. (2006), la percepción de riesgo de los estudiantes es muy baja y, aunque los estudiantes conocen la gravedad del SIDA, casi una cuarta parte de los jóvenes cree que tiene curación y el temor que inspira esta enfermedad se encuentra muy cercano al que inspira un embarazo no deseado. La relevancia de la percepción de riesgo en la prevención del SIDA es tal que algunos estudios como el de Thompson et al. (2002) han encontrado que los únicos dos predictores de las con-

Tabla 5. Solidaridad hacia los afectados por la enfermedad

¿Conoces a alguien infectado por el VIH?(Sí)

¿Cuánto dirías que sufre un enfermo con VIH física

y socialmente? $(0-100)$

La sociedad debería ser más comprensiva y solidaria

con los enfermos con VIH (0-100)

Cuidaría de un amigo que tuviera el SIDA

Si te enteraras de que un amigo tuyo es portador del VIH:

$\mathrm{Si}$ antes de conocer a alguien te enteras de que es portador del VIH:

\begin{tabular}{lr} 
& $16,8 \%$ \\
& Media $=86,9$ \\
$(D T=15,6)$ \\
& Media $=92,0$ \\
& $(D T=14,2)$ \\
& $94,6 \%$ \\
Intentarías quedar con él para apoyarle & $74,2 \%$ \\
Evitarías verle frecuentemente & $1,1 \%$ \\
Te comportarías exactamente igual que antes & $24,7 \%$ \\
Dejarías de verle por completo & $1 \%$ \\
Evitarías conocerlo & $1,6 \%$ \\
Lo conocerías exactamente igual & $87,6 \%$ \\
Tendrías más interés en conocerlo & $5,4 \%$ \\
Alertarías a tus amigos para que se preservaran & $2,2 \%$ \\
nformarías a tus amigos para que le apoyaran & $17,3 \%$ \\
\hline
\end{tabular}


ductas de riesgo son el miedo ante la transmisión del VIH y la escasa comunicación entre los miembros de la pareja sexual. Demmer y Caroleo (2001) han encontrado que una pequeña proporción de estudiantes universitarios (17\%) creían que la amenaza del SIDA es menos grave que en el pasado y el $21 \%$ indicaban que practicaban sexo seguro menos a menudo desde que se han introducido las nuevas terapias antirretrovirales.

Otro dato destacable en nuestro estudio es que menos de la mitad de jóvenes se ha realizado las pruebas de detección de anticuerpos. De hecho más de la mitad de estudiantes desconoce dónde se las puede realizar y muy pocos preguntan a sus parejas sexuales si se las han realizado. Respecto al uso del preservativo, sólo tuvo lugar en la mitad de las relaciones mantenidas en el mes anterior, siendo el uso especialmente bajo en las relaciones orales y anales, que sin embargo son mantenidas por un elevado porcentaje de los estudiantes. El bajo índice de uso del preservativo en las relaciones orales y anales también ha sido destacado por otros autores como Gurman y Borzekowski (2004). Esto indica que aunque en nuestro trabajo, sólo el $29 \%$ de los estudiantes eran conscientes de haber realizado conductas de riesgo, un porcentaje casi idéntico al informado por Planes et al. (2002) también en nuestro país, el grado de conciencia de riesgo está muy por debajo del grado de riesgo real que se deriva de las prácticas de los estudiantes.

A pesar de que el grado de confianza en el preservativo como método fiable para la prevención del SIDA es alto (a diferencia de lo que hallaron Duncan et al. en su trabajo del año 2002), de que su grado de autoeficacia para su uso es elevado y de que los estudiantes consideran que este uso está bien valorado en su entorno social, una gran mayoría de ellos encuentran barreras o dificultades para su utilización, destacando en este sentido la disminución de la sensibilidad, el miedo a su rotura y la interrupción de la relación. Quizás estas barreras lleven a que los estudiantes tiendan a sustituir el preservativo por otro método anticonceptivo cuando la relación se vuelve estable, tendencia que puede ser arriesgada teniendo en cuenta que un $11 \%$ de los estudiantes reconocen haber sido infieles en alguna ocasión a su pareja estable y de los que lo hicieron un 36\% manifestaron no haber utilizado el preservativo en esa relación esporádica.

En este sentido, numerosos estudios como el de Winfield y Whaley (2002) han destacado la importancia que tienen para la prevención del SIDA las creencias relacionadas con los beneficios y las barreras percibidas para el uso del condón, así como la autoeficacia (Burns y Di1lon, 2005) y las normas sociales percibidas (Scholly et al, 2005).

A pesar de que los datos encontrados en nuestro estudio evidencian la necesidad urgente de realizar intervenciones preventivas entre nuestros jóvenes, sí que podemos estar bastante satisfechos en lo que se refiere a sus actitudes hacia los enfermos por VIH. Aunque son muy pocos los jóvenes que conocen a alguna persona infectada por el VIH, lo que refleja sin duda el ocultamiento y estigma asociado a esta enfermedad (Ballester, 2005), el grado de sufrimiento que se atribuye a estos enfermos es alto y son una gran mayoría los estudiantes que consideran que la sociedad debería ser más solidaria con estos enfermos y los que estarían dispuestos a cuidar y apoyar a un amigo que padeciera esta enfermedad.

En resumen, la investigación realizada sobre la prevención del SIDA en el ámbito universitario nos está informando de que estamos ante un colectivo que no ha sido suficientemente atendido en las primeras décadas del SIDA. La prevalencia de las conductas de riesgo entre los estudiantes universitarios es alta, su percepción de riesgo no es lo suficientemente realista especialmente a partir de la introducción de nuevos fármacos de máxima eficacia que pueden transmitir la impresión errónea de que actualmente el SIDA no es una enfermedad tan grave. Y ni siquiera la información, que presuponíamos alta entre el colectivo universitario, es del todo adecuada con la existencia de importantes lagunas referidas a conductas de riesgo. El hecho de que estemos hablando de personas jóvenes, en pleno desarrollo sexual, con relaciones a menudo poco estables o más bien esporádicas, mayoritariamente heterosexuales con la escasa percepción de riesgo que ello conlleva y con un alto consumo de alcohol o 
de otras drogas hace que los estudiantes universitarios sean un blanco fácil para el VIH y que por tanto, sea urgente realizar intervenciones preventivas en este colectivo.

\section{REFERENCIAS}

Allison, E., y Albarracín, D. (2007). Nature, decay, and spiraling of the effects of fear-inducing arguments and HIV counseling and testing: a meta-analysis of the short- and long-term outcomes of HIV-prevention intervention. Health Psychological Association, 26, 496506.

Ballester, R. (2005). Aportaciones desde la Psicología al tratamiento de las personas con Infección por VIH. Revista de Psicopatología y Psicología Clínica, 10 , 53-69.

Ballester, R., Gil, M.D., Edo, M.T., y Giménez, C. (2007). Evolución y diferencias de género en la intención de conducta respecto a la prevención del VIH en jóvenes universitarios: años 1999-2006. X Congreso Nacional sobre el SIDA. San Sebastián, 6-8 junio (p. 183).

Ballester, R., Gil, M.D., Edo, M.T., y Ruiz, E. (2007). Autoeficacia de los jóvenes universitarios en el uso del preservativo: diferencias de género. $\mathrm{X}$ Congreso Nacional sobre el SIDA. San Sebastián, 6-8 junio (p. 188).

Ballester, R., Gil M.D., y Giménez, C. (2007a). El "Cuestionario de Prevención del SIDA" (CPS): Análisis de la fiabilidad y validez. X Congreso Nacional sobre el SIDA. San Sebastián, 6-8 de junio (p. 135).

Ballester, R., Gil M.D., y Giménez, C. (2007b). Análisis psicométrico de la "Encuesta sobre el SIDA" (ENSI): fiabilidad y validez. X Congreso Nacional sobre el SIDA. San Sebastián, 6-8 de junio (p. 135).

Ballester, R., Gil, M.D., Giménez, C., Edo, M.T., y Gil, B. (2007). Orientación sexual y evolución del uso del preservativo en jóvenes universitarios: años 1999. 2006. V World Congress of Behavioural \& Cognitive Therapies. Barcelona, 11-14 julio.

Ballester, R., Gil, M.D, Guirado, M.C., y Bravo, J.M. (2004). Eficacia de un programa de prevención del SIDA dirigido a estudiantes universitarios: datos preliminares. IV Congreso de la Asociación Española de Psicología Clínica y Psicopatología. Sevilla, 25-27 marzo (p. 163).

Ballester, R., Gil, M.D., Gómez, J.J., y Edo, M.T. (1999). Niveles de información sobre el VIH-SIDA en la Universidad. V Congreso Nacional sobre el SIDA. Santiago de Compostela, 13-16 de Abril.

Bosompra, K. (2001). Determinants of condom use intentions of university students in Ghana: An application of the theory of reasoned action. Social Science and Medicine, 52, 1057-1069.
Brodbeck, J., Matter, M., y Moggi, F. (2006). Association between cannabis use and sexual risk behavior among young heterosexual adults. AIDS and Behavior, 10, 599-605.

Burns, M., y Dillon, F. (2005). AIDS Health Locus of Control, Self-Efficacy for safer sexual practices, and Future Time Orientation as predictors of condom use in African American college students. Journal of Black Psychology, 31, 172-188.

Demmer, C., y Caroleo, O. (2001). College students' perceptions of advances in HIV treatment and the need for safer sex practices. Psychological Reports, 88, 431-442.

Duncan, C., Miller, D., Borskey, E., Fomby, B., Dawson, P., y Davis. L. (2002). Barriers to safer sex practices among African American college students. Journal of the National Medical Association, 94, 944-951.

Edo, M., y Ballester, R. (2006). Estado emocional y conducta de enfermedad en pacientes con VIH/SIDA y enfermos oncológicos. Revista de Psicopatología y Psicología Clínica, 11, 79-90.

Farid, R., y Choudhry, A. (2003). Knowledge about AIDS/ HIV infection among female college students. Journal of the College of the Physicians and Surgeons, 13, 135-137.

Flores, E., Borda, M., y San Gregorio, M.A. (2006). SIDA y exclusión social: alteraciones emocionales. Revista de Psicopatología y Psicología Clínica, 11, 37-49.

Ford, C. (1995). Psychosocial correlates of contraceptive and HIV/STD preventive behaviors among college students. Dissertation Abstracts International, 55, 4590

Giménez, C., Ballester, R., Gil, M.D., y Edo, M.T. (2006). Análisis de la tendencia del uso sistemático del preservativo en jóvenes universitarios: periodo 1999. 2005. V Congreso de la Asociación Española de Psicología Clínica y Psicopatología. Valencia, 24-25 de Noviembre.

Gullette, D., y Lyons, M. (2005). Sexual sensation seeking, compulsivity and HIV risk behaviors in college students. Journal of Community Health Nursing, 22, 47-60.

Gurman, T., y Borzekowski, D. (2004). Condom use among latino college students. Journal of American College Students, 52, 169-178.

Heeren, G.A., Jemmot, J.B., Mandeya, A., y Tyler, J.C. (2007). Theory-based predictors of condom use among university students in the United States and South Africa. AIDS, Education and Prevention, 19, 1-12.

Higgins, G. (2005). The relationship between the use of alcohol and safer sex practice in college women: a qualitative study. Dissertation Abstracts International, 66, 2293.

Lindemann, D., y Brigham, T. (2003). A Guttman scale for assessing condom use skills among college students. AIDS \& Behavior, 7, 23-27. 
Mashegoane, S., Moalusi, K., Ngoepe, M., y Peltzer, K. (2004). The prediction of condom use intention among South African university students. Psychological Reports, 95, 407-417.

Piña, J.A., y Robles, S. (2005). Psicología y VIH/SIDA en México: su prevención como base en un modelo psicológico de salud biológica. Revista de Psicopatología y Psicología Clínica, 10, 71-83.

Planes, M., Gómez, A.B., Gras, M.E., Font-Mayolas, S., Cunil, M., Aymerich, M., y Soto, J. (2006). Cambios en las percepciones de riesgo frente al SIDA de los estudiantes universitarios durante la última década. Cuadernos de Medicina Psicosomática y Psiquiatría de Enlace, 76/77, 39-45.

Planes, M., Gras, S., y Soto, J. (2002). Comportamiento anticonceptivo en estudiantes universitarios y riesgo de infección con el VIH. Anuario de Psicología, 33, 97-110.

Scholly, K., Katz, A., Gascoigne, J., y Kolck, P. (2005). Using Social Norms Theory to explain perceptions and sexual health behaviors of undergraduate college students: an exploratory study. Journal of American College Health, 53, 159-166.

Thompson, S., Kyle, D., y Swan, J. (2002). Increasing condom use by undermining perceived invulnerability to HIV. AIDS Education and Prevention, 14, 505-514.

Von Sadovsky, V., Keller, M., y McKinney, K. (2002). College students's perceptions and practices of sexual activities in sexual encounters. Journal of Nursing Scholarship, 34, 133-138.

Wilkinson, A., Holahan, C., y Drane-Edmundson, E. (2002). Predicting safer sex practices: the interactive role of partner cooperation and cognitive factors. Psychology and Health, 17, 697-709.

Winfield, E., y Whaley, A. (2002). A comprehensive test of the Health Belief Model in the prediction of condom use among African American college students. Journal of Black Psychology, 28, 330-346. 